\title{
Hypoglycemia in Older People - A Less Well Recognized Risk Factor for Frailty
}

\author{
Ahmed H Abdelhafiz ${ }^{1}$, Leocadio Rodríguez-Mañas², John E. Morley ${ }^{3}$, Alan J Sinclair ${ }^{4 *}$ \\ ${ }^{1}$ Department of Elderly Medicine, Rotherham General Hospital, England, Moorgate Road, Rotherham, UK \\ ${ }^{2}$ Hospital Universitario de Getafe, Department of Geriatrics and School of Health Sciences, Universidad Europea \\ de Madrid, Spain \\ ${ }^{3}$ Divisions of Geriatric Medicine and Endocrinology, Saint Louis University School of Medicine, St. Louis, USA \\ ${ }^{4}$ Institute of Diabetes for Older People (IDOP), University of Bedfordshire, Luton LU2 8LE, UK
}

[Received November 18, 2013; Revised February 17, 2014; Accepted March 30, 2014]

\begin{abstract}
Recurrent hypoglycemia is common in older people with diabetes and is likely to be less recognized and under reported by patients and health care professionals. Hypoglycemia in this age group is associated with significant morbidities leading to both physical and cognitive dysfunction. Repeated hospital admissions due to frequent hypoglycemia are also associated with further deterioration in patients' general health. This negative impact of hypoglycemia is likely to eventually lead to frailty, disability and poor outcomes. It appears that the relationship between hypoglycemia and frailty is bidirectional and mediated through a series of influences including under nutrition. Therefore, attention should be paid to the management of under nutrition in the general elderly population by improving energy intake and maintaining muscle mass. Increasing physical activity and having a more conservative approach to glycemic targets in frail older people with diabetes may be worthwhile.
\end{abstract}

Key Words: hypoglycemia, frailty, old age

The incidence of hypoglycemia in older people $(>75$ years) with diabetes is difficult to estimate due to the limited number of clinical studies and the lack of standardization in hypoglycemia diagnosis. In addition, many hypoglycemic episodes are mild or even asymptomatic and are not likely to be reported. However, severe single episodes of hypoglycemia may result in serious acute consequences such as seizure, coma and cardiac arrhythmias. The outcome associated with these acute consequences can be particularly debilitating in older people who are at increased risk of injury, joint dislocation and bone fractures [1]. In contrast, recurrent episodes of less severe hypoglycemia are associated with significant chronic consequences leading to physical and cognitive dysfunction and eventually frailty and disability. This severe prognosis of hypoglycemia in old age appears to be under-recognized. This review investigates the incidence and the consequences of hypoglycemia and explores its relationship to frailty, its prognostic significance and therapeutic implications in older people with diabetes.

\section{Hypoglycemia}

\section{Definition}

Hypoglycemia is defined by an arbitrary plasma glucose level of $<3.9 \mathrm{mmol} / 1$ which is the level below which activation of the counter-regulatory hormone response occurs in most adult subjects [2]. However, investigators have used hypoglycemia thresholds ranging from 3 to 3.9 $\mathrm{mmol} / \mathrm{L}$ leading to variations in the estimation of its incidence [3]. In clinical practice, the severity of hypoglycemia can be defined by the ability of an

*Correspondence should be addressed to: Alan J Sinclair, Institute of Diabetes for Older People (IDOP), University of Bedfordshire, luton LU2 8LE, UK. Email: sinclair.5@btinternet.com 
individual to self-treat (mild, asymptomatic or biochemical hypoglycemia) or an episode that requires external assistance for recovery (symptomatic or severe hypoglycemia) as classified by the Workgroup of the American Diabetes Association [2] (Table 1).

Table 1. The Workgroup classification of hypoglycemic events [2].

1) Symptomatic hypoglycemia:

2) Documented symptomatic hypoglycemia:

3) Probable symptomatic hypoglycemia:

4) Asymptomatic hypoglycemia:

5) Relative hypoglycemia:
Hypoglycemic episode needing assistance of a third party.

Symptomatic hypoglycemia and plasma glucose level $\leq 3.9 \mathrm{mmol} / \mathrm{l}$.

Symptomatic hypoglycemia but not documented a plasma glucose level.

No symptoms of hypoglycemia but documented plasma glucose level $\leq 3.9 \mathrm{mmol} / \mathrm{l}$.

Symptomatic hypoglycemia but plasma glucose level is $>3.9 \mathrm{mmol} / \mathrm{l}$ *

*This represents patients with poorly controlled diabetes for long duration who may experience hypoglycemic symptoms at a higher blood glucose level than the usual threshold of $3.9 \mathrm{mmol} / \mathrm{l}$.

\section{Recognition in old age}

Although hypoglycemia in older people ( $>75$ years) with diabetes is common, its recognition can sometimes be difficult making diagnosis in this age group uncertain. For example, due to the predominance of neurological rather than autonomic symptoms, hypoglycemia may present with symptoms such as dizziness or visual disturbance resulting in misdiagnosis [4]. Another diagnostic challenge is the similarities in the clinical presentation of hypoglycemia with that of dementia where patients present with agitation, increased confusion or behavioural changes. Furthermore, symptoms of hypoglycemia tend to be less specific with increasing age. In a survey of hypoglycemia symptoms perception by older people with diabetes mean (SD) age 82.3 (3.9) years $\}$ attending an outpatient clinic, the majority of patients reported nonspecific symptoms of being generally unwell when their blood glucose level drops, which may make the recognition of hypoglycemic episodes more difficult for the health care professionals [5]. This has also been demonstrated in the Action to Control Cardiovascular Risk in Diabetes (ACCORD) study with nonspecific fatigue or weakness as the most reported symptoms of hypoglycemia experienced by the participants, mean (SD) age 62.2 (6.8) years [6]. In older people the threshold of autonomic symptoms of hypoglycemia occurs at a lower blood glucose level and cognitive dysfunction occurs at a higher level compared with younger adults. Therefore, autonomic and neurological symptoms occur almost simultaneously with little warning [7]. This is referred to as "impaired awareness of hypoglycemia" [8]. Subclinical hypoglycemia or episodes with fewer symptoms may further reduce awareness of the onset of a severe episode because the glycemic threshold has been lowered. In other words, an episode of hypoglycemia can induce further hypoglycemia, setting a vicious circle [9]. Therefore, many episodes of hypoglycemia may be unrecognised and under reported by both patients and physicians and subsequently the frequency is likely to be underestimated (Table 2).

\section{Incidence}

The frequency of hypoglycemia varies in the literature due to the differences in the definition used, the age of the populations studied and the treatment modalities implicated. There is also a paucity of data specific to older people due to the heterogeneity of this age group particularly the incidence of hypoglycemia in frail older people or those living in care homes. In a US retrospective population-based study of 19,932 Medicaid patients, aged $\geq 65$ years [10], the incidence of serious hypoglycemia was 1.23 episodes per 100 person-years for patients treated with sulfonylureas and 2.76 episodes per 100 person-years in those treated with insulin. However, the strict definition of serious hypoglycemia (an episode leading to fatal outcome or hospital admission) may have underestimated the true frequency of events. Also, data collection of this study was conducted before the publication of the evidence for the benefit of tight glycemic control in type 2 diabetes in 1998 [11]. The increasing trends in achieving tighter glycemic control 
since then has resulted in the occurrence of more hypoglycemic episodes and with insulin to be the second most common medication associated with adverse events reported to the Federal Drug Administration and a threefold increase in the reported events from 1998 to 2005 [12]. It has also been shown that insulin was the second most frequent medication associated with emergency department visits in older people $\geq 65$ years. Of the cases attributed to insulin, $95.4 \%$ were due to hypoglycemia, $24.1 \%$ involved loss of consciousness or seizure, and $25.1 \%$ required hospitalization [13]. More recently in a prospective study of DiaRegis (multicenter registry of patients with diabetes) in Germany of 3,347 patients (median age 66.1 years), the incidence of hypoglycemia of any severity was $14.1 \%$ over 12 months of follow up [14]. Although hypoglycemia incidence is difficult to accurately estimate, it is likely to be higher in older than younger people. In a prospective observational study of 3,810 patients in primary care, $11 \%$ of participants reported at least one episode of hypoglycemia of any severity in a 12 months period. Older people ( $\geq 70$ years) reported more episodes than younger $(<60$ years) people $(12.8 \%$ vs. $9.0 \%, p<0.01)$. Significant differences were also seen for symptomatic episodes without a need for help (9.2\% vs. 5.6\%) and symptomatic episodes with a need for medical assistance $(0.7 \%$ vs. $0.1 \%)$ [15]. In care homes the incidence of hypoglycemia is likely to be much higher than in the community setting reaching up to $41.9 \%$ in one study over one year period (median 2, range 1-10 episodes per patient per year) due to the higher levels of comorbidities in this population [16].

Table 2. Difficulties in recognizing hypoglycemia in older people.

\begin{tabular}{l} 
- Symptoms are non-specific. \\
- Easily misdiagnosed, e. g. stroke, vertigo or visual disturbance. \\
- Atypical presentation e.g. confusion or passive delirium. \\
- Pittle warning or unawareness of autonomic symptoms. \\
\hline
\end{tabular}

\section{Consequences}

Older people with diabetes are likely to be at a higher risk of the adverse consequences of hypoglycemia due to the increased prevalence of multiple comorbidities, under nutrition and polypharmacy compared with younger people [17]. Severe hypoglycemia may lead to serious acute vascular events such as stroke, myocardial infarction, acute cardiac failure, and ventricular arrhythmias $[1,18]$. On the other hand, the morbidity due to recurrent episodes of hypoglycemia is associated with silent and chronic complications which could lead to significant physical and cognitive dysfunction and eventually to frailty, disability and increased mortality (Table 3).

\section{Physical dysfunction}

In a Taiwanese study of 234 residents living in long term care facilities, of whom $35.5 \%$ had diabetes with a mean (SD) age 77.5 (10.9) years, hypoglycemia was associated with disability and reduced function. Functional status, measured using the 100-point Barthel Index (BI) score, was worse in patients who experienced hypoglycemia in comparison with those with no history of hypoglycemia (mean BI score 22.5 vs 38.2). Complete dependence, defined as BI score $<30$, was also more common in patients with hypoglycemia (69.2\% vs 50\%) [16]. Lower HbAlc, which may be a surrogate marker of recurrent hypoglycemia, was also associated with more functional decline in older people with diabetes. In a longitudinal cohort study of 367 community-dwelling nursing home eligible individuals with diabetes mellitus, mean (SD) age 80 (9) years, a HbA1c of $8.0 \%$ to $8.9 \%$ was associated with a lower likelihood \{relative risk (RR) 0.88, 95\% confidence interval (CI) 0.79 to 0.99$\}$ of functional decline (defined as a decline in activity of daily living score) or death than a HbA1c of $7.0 \%$ to $7.9 \%$ after 2 years of follow up independent of age, sex, race, comorbidities and baseline function. Across all outcomes (death, functional decline, and a combined outcome), participants with $\mathrm{HbAlc}$ level $<7.0 \%$ had the highest risk of poor outcomes $(1.07,0.98$ to 1.17$)$ [19]. Risk of frequent falls is also increased with recurrent hypoglycemia. In the Health, Aging, and Body Composition cohort of 446 wellfunctioning older people with diabetes, mean (SD) age 73.6 (2.7) at baseline, tight glycemic control ( $\mathrm{HbA} 1 \mathrm{c} \leq 6 \%$ vs $>8 \%$ ) in insulin users was associated with increased risk of falls \{odds ratio (OR) $4.36,95 \%$ CI 1.32 to 14.46 \} after a mean of 4.9 years of follow up. In those using oral 
hypoglycemic medications but not insulin, same level of glycemic control (HbAlc $\leq 6 \%$ vs $>8 \%$ ) was not associated with increased risk of falls (1.29, 0.65 to 2.54$)$. This may suggest that it is not the tight glycemic control that is responsible for the increased risk of falls but it is likely to be the more recurrent episodes of hypoglycemia occurring in participants receiving insulin therapy [20]. This has also been shown in a retrospective study of 111 older people with diabetes $\geq 75$ years of age who received care through Kaiser Permanente of Colorado, USA with low $\mathrm{HbA} 1 \mathrm{c} \leq 7 \%$ increasing the risk for falls (OR 2.71, $95 \%$ CI 51.10 to 6.67 ) independent of frailty status [21]. The burden of hypoglycemia leading to hospitalisation is higher in old age which may contribute to increased frailty and reduced quality of life in this age group. In a US study of a total of 33,492 patients with diabetes, average age 60 years, accidents resulting in hospital visits occurred in $5.5 \%$ in patients with hypoglycemia compared to $2.8 \%$ of patients without. Hypoglycemia was associated with significantly increased hazards for any accident \{hazard ratio (HR) $1.39,95 \%$ CI 1.21 to $1.59, \mathrm{p}<0.001\}$, accidental falls $(1.36,1.13$ to $1.65, \mathrm{p}<0.001)$ and motor vehicle accidents $(1.82,1.18$ to $2.80, \mathrm{p}=0.007)$ adjusted for baseline characteristics. In age-stratified analysis, the risk of falls was twice as high among older people, $\geq 65$ years, compared with younger individuals and hypoglycemia was significantly associated with a greater than $50 \%$ increase in the hazard of falls (adjusted HR 1.52 , CI 1.18 to 1.95 ) [22]. Hypoglycemia also increases the risk of fractures which may lead to disability and frailty. In a retrospective observational study of 361,210 Medicare covered patients with diabetes $\geq 65$ years old, patients with hypoglycemic events had a significantly higher proportion of patients with any fall-related fractures compared to patients without (5.24\% vs. $2.67 \%$, $\mathrm{p}<0.001)$. Hypoglycemic events increased the risk of falls and fractures by $70 \%$ (OR $1.7,95 \%$ CI 1.58 to 1.83 ) [23]. Hip fracture which is a direct cause of disability and frailty is also associated with hypoglycemia. In a case control study of 932 participants with diabetes and hip fracture, mean (SD) age 77.3 (7.7) years, matched for age, sex, race, duration of diabetes mellitus and comorbidities with a similar number of control subjects, participants with tighter glycemic control (HbAlc $<6 \%$ and 6.1$7.0 \%$ ) were more likely to have a hip fracture than those with $\mathrm{HbA} 1 \mathrm{c}>8 \%$ (OR 3.01, 95\% CI 2.01 to $4.51, \mathrm{P}<$ .001 ; and $2.34,1.71$ to $3.22, \mathrm{P}<.001$, respectively) [24]. In a case control study, insulin treatment was significantly associated with bone fractures in men (OR 3.20, 95\% CI 1.32 to 7.74 ) maintained after adjusting for concomitant hypoglycemic medications. At the same time, exposure to insulin in the longer term did not appear to affect bone frailty. These results suggest that insulin increases the risk of fractures through falls caused by hypoglycemic episodes but not due to any negative effects on bone metabolism [25].

Table 3. Chronic consequences of hypoglycemia in older people.

- General physical function decline.

- Reduced ability in performing activities of daily living.

- Complete dependence.

- Frequent falls.

- Increased risk of fractures including hip fracture.

- Frequent hospitalisations.

- Increased risk of vascular disease.

- Impaired cognitive function.

- Increased risk of dementia.

- Increased fear and anxiety.

- Increased social isolation.

- Behavioural changes.

- Increased panic attacks.

- Increased risk of frailty.

- Increased risk of disability.

- Increased risk of mortality.

\section{Cognitive dysfunction}

The brain is highly dependent on glucose for its metabolism and is particularly vulnerable to hypoglycemia especially in the older people. After each hypoglycemic episode major cognitive changes occur leading to post-hypoglycemic encephalopathy. Therefore, recurrent hypoglycemia may be associated with impaired cognitive function and development of dementia. In a retrospective study of 16,667 older people with diabetes, mean age 65 years, the risk of dementia increased by $26 \%$ (HR $1.26,95 \%$ CI 1.10 to 1.49 ), $80 \%$ $(1.80,1.37$ to 2.36$)$ and $94 \%(1.94,1.42$ to 2.64$)$ in patients with a history of one, two and three or more severe hypoglycemic episodes (defined as hypoglycemia needing hospital admission or emergency department visit) respectively independent of glycemic control, medications and comorbidities. The attributable risk of dementia was $2.39 \%(1.72 \%$ to $3.01 \%)$ per year in patients with compared with those with no history of hypoglycemia [26]. In an observational cohort study of 302 participants with diabetes, mean (SD) age 75.7 (4.6) years, a cross sectional association between severe hypoglycemia and cognitive function was observed. Patients with dementia (16\% of patients) or cognitive impairment (14\%) were significantly more likely to have been hospitalized with hypoglycemia than patients with 
normal cognitive function $(3.8 \%), \mathrm{p}=0.004$ [27]. A prospective association between historical hypoglycemia and cognitive decline in a subsample of the participants without dementia who were followed up was not found. However, the prospective phase of this study was limited by the small number of participants (205 patients), short duration of follow up (18 months) which may have had limited the study power to detect the association between incident hypoglycemia and cognitive function [28]. More recently, in the Edinburgh population-based cross sectional study of 1,066 participants, mean (SD) age 67.9 (4.2) years, with type 2 diabetes, self-reported history of severe hypoglycemia was associated with poorer late-life cognitive ability. Patients who reported at least one episode of severe hypoglycemia demonstrated poorer performance on tests of Verbal Fluency (34.5 vs. 37.3, P $=0.02$ ), Digit Symbol Testing (45.9 vs. 49.9, $\mathrm{P}=0.002$ ), Letter-Number Sequencing (9.1 vs. 9.8, $\mathrm{P}=0.005)$ and
Trail Making $(\mathrm{P}<0.001)$ independent of diabetes duration, vascular risk factors or vascular complications. These associations persisted after adjustment for estimated prior cognitive ability suggesting that the association may be attributable to an effect of hypoglycemia on age-related cognitive decline [29]. A linear relationship was observed between poorer general cognitive ability and increasing frequency of severe hypoglycemia over the year preceding cognitive testing supporting results of earlier studies [26, 29]. Other mental health consequences of hypoglycemia are related to changes in patients' behavior and their fear of future episodes. They are more likely to experience anxiety and panic attacks that in turn can further increase the number of episodes and may result in social isolation and negative emotional states [30].

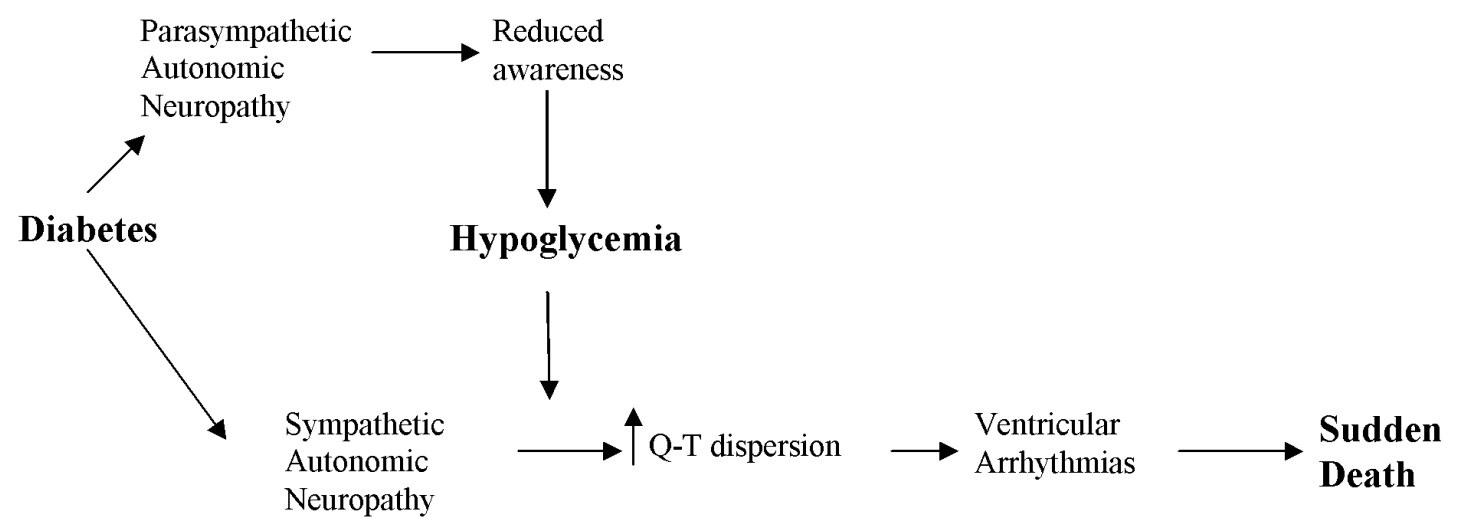

Figure 1. Dead in the Bed Syndrome and Hypoglycemia

\section{Increased mortality}

Cardiac autonomic neuropathy is a common occurrence in persons with diabetes mellitus [31, 32]. Cardiac autonomic neuropathy is associated with an increased risk of mortality which has been called the "dead-in-bed" syndrome. Nocturnal hypoglycemia interacts with sympathetic overactivity leading to an increased Q-T interval and ventricular arrhythmia which may lead to sudden death. (Figure 1) tight glycemic control has also been shown to be associated with increased mortality in the ACCORD study [33]. A more recent retrospective study of 71,092 patients with type 2 diabetes, mean (SD) age 71.0 (7.4) years, enrolled in Kaiser Permanente Northern California, has shown a U-shaped relationship between mortality and $\mathrm{HbAlc}$. Compared with mortality risk of $\mathrm{HbAlc}<0.6 \%$, mortality was lower for $\mathrm{HbA} 1 \mathrm{c}$ levels between 6.0 and $9.0 \%$, for example hazard ratio was 0.83 (95\% CI 0.76 to 0.90$)$ for $\mathrm{HbA} 1 \mathrm{c}$ 7.0-7.9\%, and higher at $\mathrm{HbAlc}>11.0 \%$ (1.3, 1.09 to 1.57$)$. Patterns were consistent across all age-groups (60-69, 70-79, and $\geq 80$ years) [34]. The U-shaped relationship has also been shown in a large UK population-based study of 47,970 patients with diabetes aged $\geq 50$ years. The hazard ratio of all-cause mortality was 1.52 (95\% CI 1.32 to 1.76$)$ at low $\mathrm{HbAlc}$ of $6.4 \%$ and 1.79 (1.56 to 2.06) at high HbAlc of $10.5 \%$. The lowest mortality risk was at $\mathrm{HbA} 1 \mathrm{c}$ of $7.5 \%$. Of interest, the mortality risk was higher in patients receiving insulin-based regimens versus those given combinations of oral agents (HR 1.49, 95\% CI 1.39 to 1.59). This suggests that hypoglycemia may have contributed to a heightened risk of mortality in patients with diabetes especially those on insulin therapy although data on the effect of hypoglycemia on mortality was 
limited [35]. However, in the ACCORD study mortality was three times higher for patients in either the conventional or intensive treatment groups who had severe hypoglycemia than in those who did not have severe hypoglycemia. Also, in the Veterans Affairs study, more than one episode of severe hypoglycemia was associated with an $88 \%$ rise in the relative risk for sudden death [36]. Therefore, the relationship between increased mortality and lower $\mathrm{HbA} 1 \mathrm{c}$ is not directly related to tight glycemic control but could be related to hypoglycemia or other factors associated with low HbAlc levels. In addition to the increased risk of recurrent hypoglycemia in those with tight glycemic control, older patients with lower $\mathrm{HbA} 1 \mathrm{c}$ levels are likely to be frailer or suffer from poor nutritional status than those with higher HbAlc levels contributing to an elevated mortality risk [37]. The U-shaped relationship of HbAlc and mortality has also been shown in non-diabetic individuals further suggesting that mortality is not related to tight glycemic control but is likely the low HbAlc level as well as recurrent hypoglycemia may just reflect under nutrition and frailty as predisposing factors to mortality [38].

\section{Frailty}

\section{Definition}

Frailty is a condition characterized by a reduction in physiological reserve and in the ability to resist physical or psychological stressors [39, 40]. Its definition was previously based on the presence of three or more phenotypes (weight loss, weakness, decreased physical activity, exhaustion and slow gait speed) [41] although it can be measured indirectly by a Frailty Index using the cumulative effects of multiple health deficits to quantify the underlying health status of the individual [42]. Measuring frailty by using the Frailty Index substantially classifies older people as frail than using the phenotypic definition approach $(22.7 \%$ vs $8.7 \%)$ [43]. A third measure is the Clinical Frailty Scale which ranks clinically distinguishable degrees of frailty into 7-point scale ranges from "very fit" to "severely frail"[44]. Finally, a rapid phenotype scale - FRAIL - has been developed and been validated to be as sensitive and specific as the other scales [45-50] (Table 4). This scale can also be used to help formulate a management plan for persons with frailty. The rate of progression of frailty increases with age and is likely due to diminishing physiologic reserve with ageing however the presence of brain pathologies including cerebrovascular, Alzheimer's and Parkinson's diseases accelerates the rate of progression [51]. The biological processes that underlie frailty are still unclear and likely to be complex and multifactorial. A recent international conference has suggested that all persons over 70 years of age should be screened for frailty with one of the rapid frailty scales [52].

Table 4. Phenotypic criteria for diagnosis of frailty The FRAIL Scale* [47].

F: Are you Fatigued?

R: Resistance: Can you walk up a flight of stairs?

A: Ambulation: Can you walk a block?

I: Do you have more than 5 Illnesses?

L: Have you Lost $5 \%$ of weight in the last 6 months?

\section{Pathogenesis}

The phenotype of frailty is thought to develop as a result of abnormalities at molecular, cellular, and physiological levels. The physiological systems believed to be associated with frailty phenotype are the cardiovascular, neuroendocrine, metabolic, immune, and nervous systems [53]. In a longitudinal cohort study of 803 participants, not frail at baseline, which examined the association between allostatic load (AL), an index of multisystem physiological dysregulation, and frailty development, each 1-unit increase in AL at baseline was associated with a $10 \%$ greater likelihood of frailty after 3 years of followup (cumulative adjusted OR 1.10, 95\% CI 1.03 to 1.19). The AL measure included 13 biomarkers from a range of physiological systems such as cardiovascular, metabolic, inflammatory, and neuroendocrine systems. This association was independent of the co-existing comorbidities or physical disabilities. The results of this study support the hypothesis that dysregulation across multiple physiological systems is associated with greater risk of frailty [54]. Frailty has also been shown to be associated with exaggerated glucose and insulin responses after 2-hour oral glucose tolerance test in individuals without diabetes which suggests that these individuals have reduced physiologic reserve and dysregulation of glucose challenge is a component of the physiologic vulnerability associated with frailty [55].

\section{Frailty and under nutrition}

Frailty is viewed as a wasting disease with weight loss being one of its criteria. In the English Longitudinal Study of Ageing which included 3,055 communitydwelling older adults aged $\geq 65$ years, increased levels of frailty has been demonstrated among those with low and very high body mass index (BMI) with lowest frailty levels in those with BMI 25-29.9 [56]. Therefore, under nutrition which is very common in older people, seems to 
be a risk factor for frailty. In the US, about $16 \%$ of elderly persons living in the community are undernourished. These figures rise to $59 \%$ in long-term care institutions and $65 \%$ in acute care hospitals [57]. Sarcopenia or muscle mass loss is a component of frailty which seems to be accelerated when diabetes is present. In a community study of 3,153 participants $\geq 65$ years of age, appendicular lean mass loss in men with diabetes was twice that of men without diabetes $(3.0 \%$ vs $1.5 \%)$ and in women with diabetes was 1.8 times that of those without diabetes $(3.4 \%$ vs $1.9 \%)$ over 4 years of follow up. The mechanisms explaining these results may be related to reduce muscle protein synthesis due to lower testosterone and insulin like growth factor 1 and increased muscle protein breakdown due to a higher rate of inflammation [58]. Diabetes also causes sarcopenia due to the catabolic effect of insulin deficiency and by increasing intramyocellular lipid accumulation [59]. In another study, older persons with type 2 diabetes had accelerated decline in leg lean mass, muscle strength, and longer sitto-stand time compared to normoglycemic controls [60]. Other factors may be related to the oral health of older people with diabetes. For example optimal nutrition may not be maintained due to poor dentition, a dry mouth, reduced taste sensation, palatability and appetite change with increasing age [61].

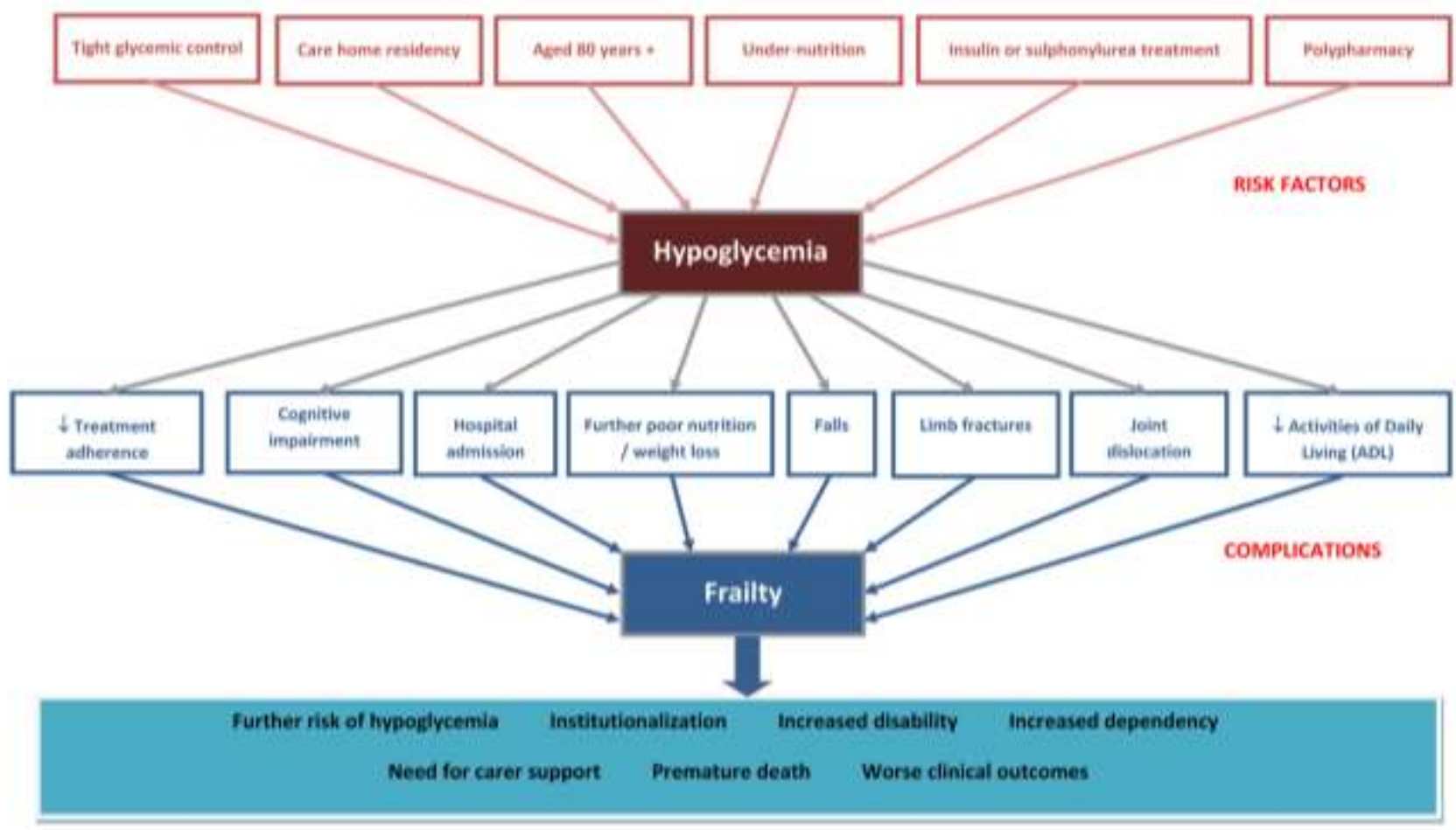

consequences

Figure 2. Hypoglycemia and Frailty - Risk Factors, Complications and Consequences.

\section{Hypoglycemia and frailty-a vicious circle}

The relationship between hypoglycemia and frailty is likely to be bidirectional. Hypoglycemia may lead to significant morbidity, disability and frailty as well as exerting a negative impact on quality of life in older people with diabetes [62]. Recurrent episodes of hypoglycemia may occur unnoticed due to the impaired autonomic response in old age which may result in subclinical accumulation of brain pathologies which may eventually lead to cognitive dysfunction and progressive physical frailty. Also frequent hospital admissions due to recurrent hypoglycemia are likely to lead to more deterioration in patients' physical and cognitive function. On the other hand frailty and dementia are risk factors for hypoglycemia setting a vicious circle. (Figure 2) It has been previously shown in a population based study of 19,932 subjects with diabetes aged $\geq 65$ years that frail 
older people (defined as oldest old, those on multiple medications and those with frequent hospitalizations) are at a higher risk for hypoglycemia. There was a trend towards increased risk of serious hypoglycemia with increasing age $(\mathrm{P}<0.05)$. Compared with persons aged 65 to 70 years, persons aged $\geq 80$ years had a higher relative risk of developing hypoglycemia (RR $1.8,95 \% \mathrm{CI}, 1.4$ to 2.3). Persons using $\geq 5$ concomitant medications were also at a higher risk for serious hypoglycemia $(1.3,1.1$ to 1.5$)$. The adjusted relative risk of serious hypoglycemia occurring in the first month after hospital discharge was 6.1 (1.4 to 26.6) compared with the risk after one year. The findings of this study suggest that older age, use of multiple medications reflecting multiple comorbidities and frequent hospitalizations which are marker of underlying frailty are associated with increased risk of hypoglycemia [10]. The risk of hypoglycemia has also been shown to be higher in patients with dementia or cognitive dysfunction. In a cross sectional study of 479,900 veterans aged $\geq 65$ years with diabetes mellitus, hypoglycemia occurred more often in participants taking insulin among those with dementia $(26.5 \%)$ and cognitive impairment (19.5\%) than in those with neither condition (14.4\%). Adjusted odds ratio for hypoglycemia in persons with dementia was 1.58 (95\% CI 1.53 to 1.62$)$ and 1.13 (1.08 to 1.18) in participants with cognitive impairment [63]. Severe hypoglycemia has also been reported in older patients with diabetes but not on any anti-diabetes medications [26, 29]. Although no explanation of this phenomenon was given by the authors, the occurrence of hypoglycemia in these patients could be related to under nutrition and frailty.

\section{Hypoglycemia and frailty-prognostic significance}

Although ageing is associated with hyperglycemic tendency due to the change in body composition with the accumulation of visceral fat increasing insulin resistance, in frail elderly there may be a tendency towards hypoglycemia due to malnutrition and comorbidities. This metabolic change towards hypoglycemia rather than hyperglycemia has been supported by the evidence of successful withdrawal of diabetes medications in nursing home older patients without deterioration in glycemic control suggesting diminished hyperglycemic or increased hypoglycemic tendency in the frail elderly [64]. The change in risk factors profile with increasing age has also been observed with other cardiovascular metabolic biomarkers such as total cholesterol and body weight and has been termed "reverse metabolic syndrome" [65]. The components of the metabolic syndrome (obesity, dyslipidemia, hypertension, hyperglycemia and insulin resistance) failed to predict mortality in the elderly and paradoxically predicted survival [65]. The mechanisms for reverse epidemiology are not yet clear but malnutrition, inflammation and functional decline may be underlying factors. It is likely that hypoglycemia acts as a marker of poor health and that it is associated with frailty and poor outcomes. For example in several diabetes studies, hypoglycemia appeared to be a marker of frailty and multiple comorbidities rather than a direct cause of adverse events. In participants included in the intensive glucose control arm of the ACCORD study, the excess mortality observed was not directly explained by the high rate of hypoglycemia but was likely attributed to unmeasured factors such as frailty [66]. In the Preterax and Diamicron Modified Release Controlled Evaluation (ADVANCE) study, hypoglycemia was strongly associated with increased risks of cardiovascular and non cardiovascular mortality. This association was independent of tight glycemic control. It is likely that hypoglycemia was related to the negative effects of the associated comorbidities and was a marker of an increased vulnerability to adverse clinical outcomes rather than a direct cause of the events. The risk estimates were markedly attenuated after adjustment for the associated comorbidities suggesting that the effects of confounding factors were substantial. It is possible that the presence of coexisting comorbidities could increase a patient's vulnerability to both hypoglycemia and an adverse outcome in the absence of a direct causal link between the two [67]. However, a recent meta-analysis suggests that severe hypoglycemia is associated with twice the risk of cardiovascular events and this association is not entirely explained by the associated comorbidities [18]. The poor outcome with hypoglycemia or low HbA1c has also been observed in the non diabetic population suggesting that factors other than tight glycemic control may explain such a relationship [38]. In the National Health and Nutrition Examination Survey III (NHANES III) of 14,099 participants without diabetes, $\mathrm{HbA} 1 \mathrm{c}<4.0 \%$ vs. $5.0-5.4 \%$ was associated with an increased risk of all-cause mortality (HR 3.73, 95\% CI 1.45 to 9.63) after 12 years of follow up [68]. Therefore, hypoglycemia as a marker of poor nutrition can also be seen as a biochemical marker of frailty in older people regardless of diabetes status. Other biochemical marker of under nutrition such as low albumin is a recognized predictor of frailty and poor outcomes. Low albumin $(<3.0 \mathrm{~g} / \mathrm{dL})$ has independently predicted the development of new onset disability at discharge amongst 885 patients $\geq 70$ years old who had been independent at hospital admission [69]. Also, albumin correlated inversely with the Frailty Index ( $\mathrm{r}-$ $0.545, \mathrm{P}<0.01)$ in another study [70]. Therefore, frailty or decline in functional reserve may be an intermediate stage between poor nutrition, manifested by low albumin and recurrent hypoglycemia, and poor outcomes in older people (Figure 2). 
Table 5: Risk factors for hypoglycemia [15, 74].

$\begin{array}{ll}\text { - } & \text { Old age } \\ \text { - } & \text { Malnutrition } \\ \text { - } & \text { Acute illness } \\ \text { - } & \text { Insulin or sulfonylurea therapy } \\ \text { - } & \text { History of hypoglycaemia } \\ \text { - } & \text { Care home residency } \\ \text { - } & \text { Recent hospitalisation } \\ \text { - } & \text { Hypoglycaemia unawareness } \\ \text { - } & \text { Blunted counter regulatory responses } \\ \text { - } & \text { Chronic hepatic or renal dysfunction } \\ \text { - } & \text { Stroke or transient ischaemic attack } \\ \text { - } & \text { Multiple medications }(\geq 5) \\ \text { - } & \text { Dementia } \\ \text { - } & \text { Depression } \\ \text { - } & \text { Heart failure } \\ \text { - } \quad \text { Social isolation }\end{array}$

\section{Hypoglycemia and frailty-therapeutic implications}

Glycaemic targets should be individualized taking into consideration individuals' overall health and life expectancy. A comfortable day to day target of a random blood glucose between $>4$ but $<15 \mathrm{mmol} / \mathrm{L}$ has been suggested to be suitable to avoid the development of symptoms as blood glucose outside this range is likely to be symptomatic, although the threshold of $>4 \mathrm{mmol} / 1$ seems too low [71]. Maintaining blood glucose in a "comfort zone" especially in frail patients may insure "comfort care" and avoid extreme blood glucose levels in order to maintain mental function and general well-being [72]. Long term targets are based on HbA1c which should be around $53 \mathrm{mmol} / \mathrm{mol}(7 \%)$ - for the fit elderly in the community with new onset diabetes similar to younger peoples' target. However, for older people with established cardiovascular disease and long standing diabetes a safer target around $58 \mathrm{mmol} / \mathrm{mol}(7.5 \%)$ is appropriate [73]. In frail older people with multiple comorbidities a target greater than $64 \mathrm{mmol} / \mathrm{mol}(8 \%)$ is suitable, especially if they are using insulin. Targets could be even higher for more frail elderly especially those in nursing homes with a very short life expectancy where day to day glucose levels are more important than the long term $\mathrm{HbA} 1 \mathrm{c}$ value to avoid symptoms and preserve quality of life [73]. Avoiding hypoglycemia should be at the core of therapeutic goals to help stop the downhill spiral of hypoglycemia-frailty vicious circle. The increased risk of hypoglycemia in older people is not only related to tight glycemic control but also to the overall health condition and associated multiple comorbidities [17]. Therefore these high-risk patients should be recognized by health care professionals and glycemic control should be carefully monitored $[15,74]$ (Table 5). Medications should be reviewed to switch patients taking longer acting sulfonylureas into shorter acting agents. In insulin treated patients, long acting insulin analogues may be a good option as they reduce the risk of hypoglycemia. Patients who have erratic eating patterns and unpredictable caloric intake could be managed with a regime where short acting insulin analogues are administered only after meal consumption, thus preventing insulin-induced hypoglycemia if a meal is missed or only partly consumed.

\section{Conclusion}

Hypoglycemia in older people with diabetes is likely to be under-recognized due to the non-specific symptoms in this age group. Also, patients who have lost the warning symptoms of hypoglycemia are vulnerable as they may not be able to recognize a hypoglycemic episode. Equally, those who cannot communicate their feelings to their carers because of cognitive impairment are at similar risk. Therefore hypoglycemia in the elderly is likely to be under-reported. Recurrent hypoglycemia is associated with significant morbidities leading to physical and cognitive dysfunction and eventually frailty and disability. The relationship between frailty and hypoglycemia appears to be dependent on multiple associated factors and in some cases is independent of diabetes status. The association of hypoglycemia and mortality is likely to be due to coexisting multiple comorbidities and frailty rather than a direct causal link between the two. Therefore, more attention should be focused on optimal management of under-nutrition in the general elderly population by improving energy intake and maintaining muscle mass and by increasing physical activity and a more conservative approach to glycemic targets in a frail population with diabetes.

\section{Key points}

Hypoglycemia in older people with diabetes is less recognized and under reported.

- In this age group, hypoglycemia is associated with significant morbidities of reduced physical and cognitive function leading to frailty and disability. 
- Hypoglycemia occurs in frail under-nourished older people regardless of diabetes status.

- Targets in frail older people with diabetes should focus on short-term day to day blood glucose levels to avoid hypoglycemia, rather than basing diabetes care around a long term HbA1c strategy.

\section{References}

[1] McAulay V, Frier BM (2009). Hypoglycemia. In Diabetes in Old Age. Sinclair A J, Eds. Chichester, UK, John Wiley and Sons. p. 287-310.

[2] Workgroup on Hypoglycemia, American Diabetes Association, Defining and reporting hypoglycemia in diabetes: a report from the American Diabetes Association Workgroup on Hypoglycemia (2005). Diabetes Care, 28: 1245-1249.

[3] Fatourechi MM, Kudvay C, Murad MH, Elamin MB, Tabini CC, Montori VM (2009). Clinical review: Hypoglycemia with intensive insulin therapy: a systematic review and meta-analyses of randomized trials of continuous subcutaneous insulin infusion versus multiple daily injections, J Clin Endocrinol Metab, 94: 729-740.

[4] Jaap A, Jones G, McCrimmon R, Deary IJ, Frier BM (1998). Perceived symptoms of hypoglycemia in elderly type 2 diabetic patients treated with insulin. Diabet Med, 15: 398-401.

[5] Abdelhafiz AH, Bailey C, Loo BE, Sinclair A (2013). Hypoglycemic symptoms and hypoglycemia threshold in older people with diabetes-a patient perspective. JNHA, 17: 899-902

[6] Bonds DE, Miller ME, Dudl J, Feinglos M, Ismail-Beigi F, Malozowski S, Seaquist E, Simmons DL, Sood A (2012). Severe hypoglycemia symptoms, antecedent behaviors, immediate consequences and association with glycemia medication usage: Secondary analysis of the ACCORD clinical trial data. BMC Endocrine Disorders, 12:5.

[7] Matyka K, Evans M, Lomas J, Cranston I, Macdonald I, Amiel SA (1997). Altered hierarchy of protective responses against severe hypoglycemia in normal aging in healthy men. Diabetes Care, 20: 135-141.

[8] Graveling AJ, Frier BM (2010). Impaired awareness of hypoglycaemia: a review. Diab Metab, 36: S64-S74.

[9] Cryer PE (2005). Mechanisms of hypoglycemiaassociated autonomic failure and its component syndromes in diabetes. Diabetes, 54: 3592-3601.

[10] Shorr RI, Ray WA, Daugherty JR, Griffin MR (1997). Incidence and risk factors for serious hypoglycemia in older persons using insulin or sulfonylureas. Arch Intern Med, 157: 1681-1686.

[11] Intensive blood-glucose control with sulphonylureas or insulin compared with conventional treatment and risk of complications in patients with type 2 diabetes (UKPDS 33). UK Prospective Diabetes Study (UKPDS) Group (1998). Lancet, 352: 837-853.
[12] Moore TJ, Cohen MR, Furberg CD (2007). Serious adverse drug events reported to the Food and Drug Administration, 1998-2005. Arch Intern Med, 167: 1752-1759.

[13] Budnitz DS, Shehab N, Kegler SR, Richards CL (2007). Medication use leading to emergency department visits for adverse drug events in older adults. Ann Intern Med, 147: 755-765.

[14] Tschöpe D, Bramlage P, Binz C, Krekler M, Deeg E, Gitt AK (2012). Incidence and Predictors of Hypoglycemia in Type 2 Diabetes. BMC Endocr Disord, doi: 10.1186/1472-6823-12-23.

[15] Bramlage P, Gitt AK, Binz C, Krekler M, Deeg E, Tschöpe D (2012). Oral antidiabetic treatment in type-2 diabetes in the elderly: balancing the need for glucose control and the risk of hypoglycemia. Cardiovascular Diabetology, doi: 10.1186/1472-6823-12-23.

[16] Chen LK, Lin MH, Lai HY, Hwang SJ (2008). Care of patients with diabetes mellitus in long-term care facilities in Taiwan: diagnosis, glycemic control, hypoglycemia, and functional status. J Am Geriatr Soc, 56: 1975-1976.

[17] Abdelhafiz AH, Sinclair AJ (2009). Hypoglycemia in residential care homes. Br J Gen Pract, 59: 49-50.

[18] Goto A, Arah OA, Goto M, Terauchi Y, Noda M (2013). Severe hypoglycemia and cardiovascular disease: systematic review and meta-analysis with bias analysis. BMJ, 347:f4533 doi: 10.1136/bmj.f4533.

[19] Yau CK, Eng C, Cenzer IS, Boscardin WJ, RiceTrumble K, Lee SJ (2012). Glycosylated Hemoglobin and Functional Decline in Community-Dwelling Nursing Home-Eligible Elderly Adults with Diabetes Mellitus. J Am Geriatr Soc, 60: 1215-1221.

[20] Schwartz AV, Vittinghoff E, Sellmeyer DE, et al (2008). Diabetes-Related Complications, Glycemic Control, and Falls in Older Adults. Diabetes Care, 31: 391-396.

[21] Nelson JM, Dufraux K, Cook PF (2007). The Relationship Between Glycemic Control and Falls in Older Adults. J Am Geriatr Soc, 55: 2041-2044.

[22] Signorovitch JE, Macaulay D, Diener M, Yan Y, Wu EQ, Gruenberger JB, Frier BM (2013). Hypoglycemia and accident risk in people with type 2 diabetes mellitus treated with non-insulin anti-diabetes drugs. Diab Obes Metab, 15: 335-341.

[23] Johnston SS, Conner C, Aagren M, Ruiz K, Bouchard J (2012). Association between hypoglycemic events and fall-related fractures in Medicare-covered patients with type 2 diabetes. Diab Obes Metab, 14: 634-643.

[24] Puar TH, Khoo JJ, Cho LW, Xu Y, Chen YT, Chuo AM, Poh CB, Ng JM (2012). Association Between Glycemic Control and Hip Fracture. J Am Geriatr Soc, 60: 14931497.

[25] Nonami M, Cresci B, Colombini A, Pala L, Balzi D, Gori F, Chiasserini V, Marchionni N, Rotella CM, Mannucci E (2008). Bone Fractures and Hypoglycemic Treatment in Type 2 Diabetic Patients. Diabetes Care, 31: 199-203.

[26] Whitmer RA, Karter AJ, Yaffe K, Quesenberry CP Jr, Selby JV (2009). Hypoglycemic episodes and risk of 
dementia in older patients with type 2 diabetes mellitus. JAMA, 301: 1565-1572.

[27] Bruce DG, Davis WA, Casey GP, Starkstein SE, Clarnette RM, Foster JK, Almeida OP, Davis TM (2008). Predictors of cognitive impairment and dementia in older people with diabetes. Diabetologia, 51: 241248.

[28] Bruce DG, Davis WA, Casey GP, et al (2009). Severe hypoglycaemia and cognitive impairment in older patients with diabetes: the Fremantle Diabetes Study. Diabetologia, 52: 1808-1815.

[29] Aung PP, Strachan MWJ, Frier BM, Butcher I, Deary IJ, Price JF (2012). Severe hypoglycemia and late-life cognitive ability in older people with Type 2 diabetes: the Edinburgh Type 2 Diabetes Study. Diabet. Med, 29: 328-336.

[30] Wild D, von Maltzahn R, Brohan E, Christensen T,Clauson P, Gonder-Frederick L (2007). A critical review of the literature on fear of hypoglycemia in diabetes: Implications for diabetes management and patient education. Patient Educ Couns, 68: 10-15.

[31] Morley JE (2013). Syncope. J Am Med Dir Assoc, 14: 311-312.

[32] Vinik AI, Maser RE, Ziegler D (2011). Autonomic imbalance: Prophet of doom or scope for hope? Diabet Med, 28: 643-651.

[33] Gerstein HC, Miller ME, Byington RP, et al (2008). Action to Control Cardiovascular Risk in Diabetes Study Group. Effects of intensive glucose lowering in type 2 diabetes. N Engl J Med, 358: 2545-2559.

[34] Huang ES, Liu JY, Moffet HH, John PM, Karter AJ (2011). Glycemic Control, Complications and Death in Older Diabetic Patients. Diabetes Care, 34: 1329-1336.

[35] Currie CJ, Peters JR, Tynan A, Evans M, Heine RJ,Bracco OL, Zagar T, Poole CD (2010). Survival as a function of $\mathrm{HbA} 1 \mathrm{c}$ in people with type 2 diabetes: a retrospective cohort study. Lancet, 375: 481-489.

[36] Ray KK, Seshasai SR, Wijesuriya S, Sivakumaran R, Nethercott S, Preiss D, Erqou S, Sattar N (2009). Effect of intensive control of glucose on cardiovascular outcomes and death in patients with diabetes mellitus: a meta-analysis of randomised controlled trials. Lancet, 373: 1765-1772.

[37] Kim TN, Park MS, Yang SJ, et al (2010). Prevalence and determinant factors of sarcopenia in patients with type 2 diabetes: the Korean Sarcopenic Obesity Study (KSOS). Diabetes Care, 33: 1497-1499.

[38] Selvin E, SteffesMW, Zhu H, et al (2010). Glycated hemoglobin, diabetes and cardiovascular risk in nondiabetic adults. N Engl J Med, 362: 800-811.

[39] Bergman H, Ferrucci L, Guralnik J Hogan DB, Hummel S, Karunananthan S, Wolfson C (2009). Frailty: An emerging research and clinical paradigm-issues and controversies. J Gerontol A Biol Sci Med Sci, 62A: 731737.

[40] Morley JE (2009). Developing novel therapeutic approaches to frailty. Curr Pharm Des, 15: 3384-3395.

[41] Fried LP, Tangen CM, Walston J Newman AB, Hirsch C, Gottdiener J, Seeman T, Tracy R, Kop WJ, Burke G, McBurnie MA (2001). Frailty in older adults: Evidence for a phenotype. J Gerontol A Biol Sci Med Sci, 56A: M146-M56.

[42] Peña FG, Theou O, Wallace L, et al (2014). Comparison of alternate scoring of variables on the performance of the frailty index. BMC Geriatrics, 14:25 doi: 10.1186/1471-2318-14-25.

[43] Song X, Mitnitski A, Rockwood K (2010). Prevalence and 10-Year Outcomes of Frailty in Older Adults in Relation to Deficit Accumulation. J Am Geriatr Soc, 58: 681-687.

[44] Rockwood K, Song X, MacKnight C, Bergman H, Hogan DB, McDowell I, Mitnitski A (2005). A global clinical measure of fitness and frailty in elderly people. Can Med Assoc J, 173: 489-495.

[45] Woo J, Leung J, Morley JE (2012). Comparison of frailty indicators based on clinical phenotype and the multiple deficit approach in predicitng mortality and physical limitation. J Am Geriatr Soc, 60: 1478-1486.

[46] Abellan van Kan G, Rolland YM, Morley JE, Vellas B (2008). Frailty: Toward a clinical definition. J Am Med Dir Assoc, 9: 71-72.

[47] Morley JE, Malmstrom TK, Miller DK (2012). A simple frailty questionnaire (FRAIL) predicts outcomes in middle aged African Americans. J Nutr Health Aging, 16: 601-608.

[48] Ravindrarajah R, Lee DM, Pye SR, et al (2013). The ability of three different models of frailty to predict allcause mortality: Results from the European Male Aging Study (EMAS). Arch Gerontol Geriatr, 57: 360-368.

[49] Hyde Z, Flicker L, Almeida OP, Hankey GJ, McCaul KA, Chubb SA, Yeap BB (2010). Low free testosterone predicts frailty in older men: The health in men study. $\mathbf{J}$ Clin Endocrinol Metab, 95: 3165-3172.

[50] Lopez D, Flicker L, Dobson A (2012). Validation of the frail scale in a cohort of older Australian women. J Am Geriatr Soc, 60: 171-173.

[51] Buchman AS, Yu L, Wilson RS, Schneider JA, Bennett DA (2013). Association of brain pathology with the progression of frailty in older adults. Neurology, 80: 17.

[52] Morley JE, Vellas B, van Kan GA, et al (2013). Frailty consensus: A call to action. J Am Med Dir Assoc, 14: 392-397.

[53] Walston J, Hadley EC, Ferrucci L, et al (2006). Research agenda for frailty in older adults: Toward a better understanding of physiology and etiology: Summary from the American Geriatrics Society/National Institute on Aging Research Conference on Frailty in Older Adults. J Am Geriatr Soc, 54: 991-1001.

[54] Gruenewald TL, Seeman TE, Karlamangla AS, Sarkisian CA (2009). Allostatic Load and Frailty in Older Adults. J Am Geriatr Soc, 57: 1525-1531.

[55] Kalyani RR, Varadhan R, Weiss CO, Fried LP, Cappola AR (2012). Frailty Status and Altered Glucose-Insulin Dynamics. J Gerontol A Biol Sci Med Sci, 67: 13001306.

[56] Hubbard RE, Lang IA, Llewellyn DJ, Rockwood K (2010). Frailty, Body Mass Index, and Abdominal Obesity in Older People. J Gerontol ABiol Sci Med Sci, 65A: 377-381. 
[57] Beers MH, Berkow R (eds). The Merck manual of geriatrics. Medical services, USMEDSA, USHH. Section 1. Nutritional disorders. Malnutrition; 20002004.

[58] Lee JSW, Auyeung TW, Leung J, Kwok T, Leung PC, Woo J (2010). The effect of diabetes mellitus on ageassociated lean mass loss in 3153 older adults. Diabet Med, 27: 1366-1371.

[59] Landi F, Onder G, Bernabei R (2013). Sarcopenia and diabetes: Two sides of the same coin. J Am Med Dir Assoc, 14: 540-541.

[60] Leenders M, Verdijk LB, van der Hoeven L, Adam JJ, van Kranenburg J, Nilwik R, van Loon LJ (2013). Patients with type 2 diabetes show a greater decline in muscle mass, muscle strength, and functional capacity with aging. J Am Med Dir Assoc, 14: 585-592.

[61] Rizvi AA (2009). Nutritional challenges in the elderly with diabetes. Intern J Diabetes Mellitus, 1: 26-31.

[62] Zhang Y, Wieffer H, Modha R (2010). The Burden of Hypoglycemia in Type 2 Diabetes: A Systematic Review of Patient and Economic Perspectives. JCOM, 17: 547-57.

[63] Feil DG, Rajan M, Soroka O, Tseng CL, Miller DR, Pogach LM (2011). Risk of Hypoglycemia in Older Veterans with Dementia and Cognitive Impairment: Implications for Practice and Policy. J Am Geriatr Soc, 59: 2263-72.

[64] Sjoblom P, Tengblad A, Lofgren UB, Lannering C, Anderberg N, Rosenqvist U, Mölstad S, Ostgren CJ (2008). Can diabetes medication be reduced in elderly patients? An observational study of diabetes drug withdrawal in nursing home patients with tight glycaemic control. Diab Res Clin Pract, 82: 197-202.

[65] Vischer UM, Safar ME, Safar H, Iaria P, Le Dudal K, Henry O, Herrmann FR, Ducimetière P, Blacher J (2009). Cardiometabolic determinants of mortality in a geriatirc population: Is there a "reverse metabolic syndrome"? Diab Metab, 35: 108-14.

[66] Bonds DE, Miller ME, Bergenstal RM, Buse JB, Byington RP, Cutler JA, Dudl RJ, Ismail-Beigi F, Kimel AR, Hoogwerf B, Horowitz KR, Savage PJ, Seaquist ER, Simmons DL, Sivitz WI, Speril-Hillen JM, Sweeney ME (2010). The association between symptomatic, severe hypoglycaemia and mortality in type 2 diabetes: retrospective epidemiological analysis of the ACCORD study. BMJ, 340: b4909. doi:10.1136/bmj.b4909.

[67] Zoungas S, Patel A, Chalmers J, de Galan BE, Li Q, Billot L, Woodward M, Ninomiya T, Neal B, MacMahon S, Grobbee DE, Kengne AP, Marre M, Heller S (2010). Severe Hypoglycemia and Risks of Vascular Events and Death. N Engl J Med, 363: 14101418.

[68] Carson AP, Fox CS, McGuire DK, Levitan EB, Laclaustra M, Mann DM, Muntner P (2010). Low Hemoglobin A1c and Risk of All-Cause Mortality Among US Adults Without Diabetes. Circ Cardiovasc Qual Outcomes, 3: 661-667.

[69] Mehta KM, Pierluissi E, Boscardin WJ, Kirby KA, Walter LC, Chren MM, Palmer RM, Counsell SR, Landefeld CS (2011). A Clinical Index to Stratify Hospitalized Older Adults According to Risk for NewOnset Disability. J Am Geriatr Soci, 59: 1206-1216.

[70] Hubbard RE, O’Mahony MS, Savva GM, Calver BL, Woodhouse KW (2009). Inflammation and frailty measures in older people. J Cell Mol Med, 13: 31033109.

[71] Cox DJ, Kovatchev BP, Gonder-Frederick LA, Summers KH, McCall A, Grimm KJ, Clarke WL (2005). Relationships between hyperglycemia and cognitive performance among adults with type 1 and type 2 diabetes. Diabetes Care, 28: 71-77.

[72] Lee SJ, Eng C (2011). Goals of Glycemic Control in Frail Older Patients With Diabetes. JAMA, 305: 13501351.

[73] Sinclair A, Morley JE, Rodriguez-Mañas L, Paolisso G, Bayer T, Zeyfang A, Bourdel-Marchasson I, Vischer U, Woo J, Chapman I, Dunning T, Meneilly G, RodriguezSaldana J, Gutierrez Robledo LM, Cukierman-Yaffe T, Gadsby R, Schernthaner G, Lorig K (2012). Diabetes mellitus in older people: Position statement on behalf of the International Association of Gerontology and Geriatrics (IAGG), the European Diabetes Working Party for Older People (EDWPOP), and the International Task Force of Experts in Diabetes. J Am Med Dir Assoc, 13: 497-502.

[74] Chelliah A, Burge MR (2004). Hypoglycemia in elderly patients with diabetes mellitus: causes and strategies for prevention. Drugs Aging, 21: 511-530. 